\section{CPS-073 FOLLOW-UP OF RECOMMENDATIONS ON DOSE ADJUSTMENT OF CEFTOLOZANE/TAZOBACTAM IN RENAL FAILURE}

${ }^{1} \mathrm{M}$ Rodriguez-Reyes*, 'E López-Suñé, ${ }^{1} \mathrm{E}$ Calvo-Cidoncha, ${ }^{1} \mathrm{M}$ Tuset-Creus, ${ }^{1} \mathrm{C}$ Codina-Jané, ${ }^{2}$ A Soriano-Viladomiu. ${ }^{1}$ Hospital Clinic, Pharmacy Department, Barcelona, Spain; ${ }^{2}$ Hospital Clínic, Infectious Diseases Department, Barcelona, Spain

\subsection{6/ejhpharm-2019-eahpconf.222}

Background Ceftolozane/tazobactam is a novel antibiotic commonly used in infections by gram-negative bacteria resistant to conventional antibiotics. Drug-dosing errors are common in patients with renal impairment and can cause adverse effects or poor outcomes.

Purpose To determine the adequacy of ceftolozane/tazobactam dose adjustment according to renal function in hospitalised patients.

Material and methods Retrospective observational study in a third-level hospital involving patients treated with ceftolozane/ tazobactam from January to August 2018. Variables collected: sex, age, creatinine clearance $(\mathrm{CrCl})$, medical/critical care unit, type of infection, microorganisms isolated, type of therapy (empiric or targeted), posology, treatment duration, effectiveness of treatment (microbiological and/or clinical cure) and dosage adequacy. Underdosing was defined as any dose lower than the Summary of Product Characteristics recommended dose (based on $\mathrm{CrCl}$ ) and overdosing was the opposite. For pneumonia (off-label), a double dose was considered according to the Stanford Health Care Antimicrobial Dosing Reference Guide.

Results Forty-six patients were included: $65.2 \%$ were male, mean age was $65.4 \pm 16.2$ years and mean $\mathrm{CrCl}$ was 61.8 $\pm 30.6 \mathrm{~mL} / \mathrm{min}$. At the beginning of treatment, $41.3 \% \mathrm{had}$ $\mathrm{CrCl}<60 \mathrm{~mL} / \mathrm{min}$. Sixteen patients $(34.8 \%)$ were admitted to the intensive care unit. Main infection sites were: respiratory $(43.5 \%)$, urinary $(30.4 \%)$ and intra-abdominal (15.2\%). Therapy was basically targeted $(73.9 \%)$ and the most common isolated pathogen was multidrug-resistant Pseudomonas aeruginosa (90.9\%). Average treatment duration was 8.4 days.

Evaluation at first day of therapy showed that 29 patients $(63.0 \%)$ received an inappropriate dosage, 18 (39.1\%) were underdosed and $11(23.9 \%)$ were overdosed. During treatment, 16 patients experienced a change in $\mathrm{CrCl}$ but dose was not adjusted accordingly in the majority of cases $(n=10$, $62.5 \%)$.

Patients with empiric treatment had a favourable evolution. Among patients with targeted therapy and respiratory, urinary or intra-abdominal infection $(n=30)$ treatment was effective in
$23(76.7 \%)$. Ceftolozane/tazobactam was de-escalated in two $(6.7 \%)$, changed by another antibiotic because of inefficacy in two $(6.7 \%)$ and discontinued because of poor prognosis in three $(10.0 \%)$.

Conclusion A considerable proportion of patients treated with ceftolozane/tazobactam were inappropriately dosed. Furthermore, dosage was not adapted to the changes in renal function throughout the treatment. These data highlight the importance of an adequate review of medication.

\section{REFERENCES AND/OR ACKNOWLEDGEMENTS}

No conflict of interest.

\section{CPS-074 ROLE OF THE PHARMACIST IN THE EVALUATION OF THE PRESCRIPTIVE APPROPRIATENESS IN ANTIBIOTIC THERAPY AS A 'SINGLE DOSE'}

${ }^{1} \mathrm{P}$ Sorice*, 'L Armillei, ' $\mathrm{G}$ Di Florio, ${ }^{1} \mathrm{~F}$ Gasbarri, ${ }^{2} \mathrm{~S}$ Rossetti, ${ }^{1} \mathrm{~S}$ Corridoni, ${ }^{1} \mathrm{~A}$ Costantini. ${ }^{1}$ Hospital Santo Spirito Pescara, Hospital Pharmacy, Pescara, Italy; ${ }^{2}$ Hospital Santo Spirito Pescara, Hospital Pharmacy - Trainee, Pescara, Italy

\subsection{6/ejhpharm-2019-eahpconf.223}

Background The importance of prescriptive appropriateness in antibiotic treatment derives from the need to combine the effectiveness of care with available resources, making them accessible to all.

An inappropriate prescription may cause errors that can have important consequences in both patient's health and healthcare costs. With the prescription of antibiotics, it is also important to reduce the phenomenon of resistance.

Currently, the strategies adopted to reduce errors in therapy are:

- Computerised systems for prescribing and administering therapy.

- Preparation and distribution of unit dose drugs.

- Control and validation of therapy by the clinical pharmacist.

Our hospital has been managing the unit dose system since 2005. Currently, there are 18 units under unique dose with a total of 400 beds.

Purpose The aim of this study was to evaluate the pharmacist's contribution to risk management to increase the appropriateness of antibiotic prescriptions and reduce costs.

All the therapies that have been modified following a pharmacist's report and, therefore, the degree of acceptance of notifications by the medical staff were examined.

Material and methods The analysis was carried out by extrapolating, from the prescription software, the medical prescriptions of the antibiotics during June to December 2016 and June to December 2017. We found the following discrepancies:

- Posology (dosage, administration frequency, route of administration, duration of therapy).

- Therapeutic indication.

- Pharmacological interactions.

- Instructions on how to dilute.

- Intolerances/allergies.

Results From June to December 2016, 279 inappropriate therapies were reported by the pharmacist. Of these, 19\% (53) were modified by the doctor.

In the period June to December 2017, 430 reports were introduced, of which $26.51 \%$ (114) were modified by the doctor. 